\title{
The role of physical activity during pregnancy in determining maternal and foetal outcomes
}

\author{
JT Pearson, ${ }^{1}$ BSc (Med) (Hons) (Exerc Sci) (Biokinetics); \\ ED Watson, ${ }^{2,3}$ MSpSc; E V Lambert, ${ }^{1}$ PhD; L K Micklesfield, ${ }^{2} \mathrm{PhD}$ \\ ${ }^{1}$ Division of Exercise Science \& Sports Medicine, Department of Human
Biology, Faculty of Health Sciences, University of Cape Town, South Africa \\ ${ }^{2}$ MRC/WITS Developmental Pathways for Health Research Unit, Department \\ of Paediatrics, Faculty of Health Sciences, University of the Witwatersrand, \\ Johannesburg, Africa \\ ${ }^{3}$ Centre for Exercise Science and Sports Medicine, School of Therapeutic \\ Sciences, Faculty of Health Sciences, University of Witwatersrand, \\ Johannesburg, South Africa
}

Corresponding author: LK Micklesfield (lisa.micklesfield@uct.ac.za)

Background. A woman's health status prior to, and during, pregnancy has been shown to influence maternal and foetal health outcomes, and therefore healthy behaviours should be encouraged to optimise weight gain during pregnancy.

Discussion. Pre-gravid overweight and obesity, and excessive weight gain during pregnancy, are associated with several adverse pregnancy outcomes for the mother and the foetus. Despite the fact that physical activity during pregnancy reduces the risk of excessive gestational weight gain, physical activity levels typically decline during pregnancy. Several factors have been shown to influence physical activity during pregnancy, and preliminary data is available on identifying these factors in South African women.

Conclusion. Very little is known about physical activity patterns in pregnant South African women in whom overweight and obesity is prevalent. Examining these patterns, as well as the barriers and facilitators of physical activity in this population will assist in informing future interventions.

Keywords. South Africa, obesity, barriers, interventions

S Afr J Sports Med 2015;27(4):93-96. DOI:10.17159/2078-516X/2015/ v27i4a 440

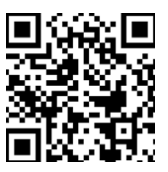

In South Africa (SA), as the population undergoes economic transition and HIV/AIDS mortality rates become more controlled, non-communicable diseases (NCDs) are emerging as one of the major healthcare burdens facing the country. ${ }^{[1]}$ In fact, this rise in NCDs, coupled with maternal and infant mortality rates, form two of the four main threats to the health of South Africans. ${ }^{[2]}$ NCDs are said to account for $21 \%$ of life years lost in SA. ${ }^{[1]}$ In particular, the obesity epidemic has been increasing, with the most recent statistics reporting that $61 \%$ of the SA population are reportedly overweight or obese, with the prevalence being particularly high in women. ${ }^{[2]}$

There is strong evidence for the role of physical activity (PA) in reducing the risk and burden of NCDs, ${ }^{[3]}$ which is of particular importance in South African women, who appear to be vulnerable to both weight gain and physical inactivity. ${ }^{[4]}$ For example, data from the South African Demographic and Health Survey (2003) showed that two-thirds of South African women are inactive, with physical inactivity being a major contributing factor to the increase in overweight or obesity. ${ }^{[4]}$ According to the first South African National
Health and Nutrition Examination Survey (SANHANES-1), it appears that the most significant increase in waist circumference, as well as overweight and obesity, occur between 15 and 35 years of age. As this represents childbearing age, it may be the perfect window of opportunity to intervene, not only to optimise the health of the mother but also of her offspring. By optimising health behaviours and contributing to the achievement of the World Health Organisation's (WHO) Millennium goals, this may assist in reducing child mortality and improving maternal health.

Recent preventative medicine research has focused on early life and in utero environmental factors and their effects on future health with some studies showing that, although the genetics of disease cannot be ignored, early life factors may play an important role in the risk of disease. ${ }^{[5]}$ This alludes to the fact that a woman's health status, both pre-pregnancy and during pregnancy, can significantly influence pregnancy outcomes and the health prospects of the offspring. ${ }^{[5]}$ Therefore the importance of optimising the period of pre-pregnancy and pregnancy, and encouraging healthy behaviours during this time, should be emphasised.

Despite the wealth of evidence to encourage physical activity in the prevention and management of chronic diseases, minimal research exists for the pregnant population, particularly in developing countries. It has been shown that women of lower educational and socioeconomic status are particularly vulnerable to adverse pregnancy outcomes due to limited knowledge around pregnancy and health, including physical activity recommendations. ${ }^{[6]}$ Therefore the purpose of this review is to describe the effects of overweight and obesity, and the role of physical activity, on maternal and foetal health outcomes during pregnancy. In addition, the factors that have been shown to influence physical activity participation during pregnancy have been reviewed.

\section{The burden of obesity in South Africa}

For almost 20 years South Africa has been burdened with an increasing prevalence of NCDs, such as cardiovascular disease and type 2 diabetes, overweight and obesity, and their associated disease burdens. ${ }^{[1]}$ Although physical inactivity and sub-optimal nutrition are not unique to South Africa, the country has gone through drastic economic change and urbanisation, which may have magnified these global trends. ${ }^{[2]}$

Unwin et al. suggest that urbanisation, which is associated with an increase in unhealthy dietary behaviours and increased physical inactivity, has the potential to increase the burden of NCDs on the healthcare sector. ${ }^{[7]} \mathrm{A}$ review by Mayosi et al. revealed that the burden of NCDs appears to be disproportionately higher in poor people living in urban settings. ${ }^{[1]}$ Increased urbanisation has been associated with a rise in the prevalence of hypertension, ${ }^{[7]}$ and Bourne et al. ${ }^{[8]}$ named urbanisation as one of the four independent risk factors for type 2 diabetes. Kruger et al. ${ }^{[9]}$ identified urbanisation as one of the top three predictors of increased body mass index (BMI), skinfold thickness, waist circumference and waist-to-hip ratio, all of which were associated with an increase in the prevalence of elevated blood pressure and triglycerides, as well as reduced glucose tolerance and insulin sensitivity. 
South African national data have reported that women have a higher BMI and prevalence of obesity than men, with a reported prevalence of overweight and obesity in females between $40-56 \%{ }^{[1,2,4]}$ This is noteworthy, since extensive literature has reported that being overweight or obese prior to, and during, pregnancy has adverse effects on both the mother and the foetus during pregnancy and postpartum. ${ }^{[9,10]}$

\section{Obesity and maternal outcomes}

Pre-gravid overweight or obesity has been shown to result in adverse pregnancy outcomes, such as hypertension, pre-eclampsia, gestational diabetes mellitus (GDM), perinatal mortality, macrosomia and complicated deliveries. ${ }^{[10]}$ In addition, women who are overweight or obese prior to pregnancy have a greater risk of miscarriage compared to women with a normal BMI, ${ }^{[10]}$ and may also be at an increased risk of Caesarean section delivery. ${ }^{[11]}$ Consequently, women who enter pregnancy overweight or obese tend to stay in hospital longer and incur greater financial costs around the time of delivery and postpartum. ${ }^{[10]}$ Thus it is advisable that women are at an optimal and healthy body weight prior to falling pregnant in order to improve their chances of a successful pregnancy.

Excessive gestational weight gain is associated with the development of maternal hypertension and pre-eclampsia. ${ }^{[1]}$ Globally, approximately $10 \%$ of all pregnancies are complicated by hypertension, which is a major cause of both foetal and maternal morbidity and mortality. ${ }^{[12]}$ In South Africa, the latest Saving Mothers Report cited 622 maternal deaths related to hypertensive disorders, with $55.3 \%$ from eclampsia, $28.3 \%$ from pre-eclampsia and $6.1 \%$ associated with chronic hypertension. ${ }^{[13]}$ According to this report, deaths from hypertensive disorders of pregnancy remain the most common direct cause of maternal death. ${ }^{[13]}$ As stated above, since hypertension is closely associated with overweight and obesity, as well as excessive gestational weight gain, there appears to be a need for continual education of women to reduce the risk of developing hypertension, by reducing pre-pregnancy weight and limiting weight gain during pregnancy. ${ }^{[13]}$

Similarly, women who enter pregnancy overweight or obese, or gain excessive weight during pregnancy, have an increased risk of developing GDM. ${ }^{[10]}$ Although little is known about GDM in Africa, a recent systematic review reported a prevalence ranging from $0-13.9 \%$, and an estimated $8.8 \%$ in South Africa. ${ }^{[14]}$ GDM not only increases the risk of maternal complications, but many studies have shown that children of women with GDM have a higher risk of childhood obesity and metabolic dysregulation later on in life. ${ }^{[15]}$ Certainly, in the mother, there appears to be a $40 \%$ greater risk of developing type 2 diabetes mellitus (T2DM) within 15 years of giving birth. ${ }^{[13]}$

\section{Maternal obesity and foetal outcomes}

The WHO recently described the 1000 days between conception and the child's second birthday as a critical period for determining the future risk of disease. Drake and Walker ${ }^{[16]}$ suggest that intergenerational effects may occur as a result of genetic attributes manifesting in a similar manner in both the mother and her child, adverse extrinsic environmental conditions persisting from one generation to the next, or adverse intrauterine conditions resulting in an altered maternal metabolism, which in turn provides an adverse environment for the foetus.
For example, high pre-gravid BMI and low pre-gravid insulin sensitivity are strong predictors of increased foetal growth, possibly due to the maternal insulin resistance resulting in greater availability of nutrients to the foetus. ${ }^{[9]}$ Pre-pregnancy BMI and gestational weight gain have been found to predict birth weight, ${ }^{[10]}$ with higher BMI being associated with greater risk of delivering large for gestational age infants. ${ }^{[11]}$ Several studies have shown that increased birth weight increases the risk of infant mortality and birth trauma, and is also associated with an increased risk of future overweight and obesity in the child. ${ }^{[15]}$ This, in turn, puts the child at risk of developing further complications associated with obesity, such as type 2 diabetes ${ }^{[11]}$ and cardiovascular disease ${ }^{[5]}$ later in life.

\section{The role of physical activity in pregnancy}

In a review by Min-Lee et al. ${ }^{[3]}$ physical inactivity was equated with smoking and obesity as an established NCD risk factor. Indeed, the review estimated that inactivity causes $9 \%$ of premature mortality and up to $10 \%$ of NCDs, worldwide. ${ }^{[3]}$ Likewise, in South Africa, physical inactivity has been shown to be associated with obesity. ${ }^{[4]}$ Therefore PA has an important role to play in the prevention and management of disease, and is also of particular importance during pregnancy.

A review by Gavard and Artal ${ }^{[17]}$ concluded that PA during pregnancy reduces maternal and foetal morbidities, and produces long-term benefits for both the mother and the child. Various studies have shown that PA reduces excessive gestational weight gain (GWG) as well as postpartum obesity, and reduces the likelihood of preeclampsia and gestational diabetes. ${ }^{[18]}$

Olson and Strawderman ${ }^{[19]}$ conducted a prospective cohort study in New York in which socio-demographic characteristics, exercise, food-related behaviours and breastfeeding were assessed using medical records and the responses from a mailed questionnaire. Body weight was measured at prenatal visits and one year postpartum. Weight retained and major weight gain $(4.55 \mathrm{~kg})$ at one year postpartum were the main outcomes. ${ }^{[19]}$ This study showed that physical inactivity during pregnancy is associated with excessive GWG when compared with maintaining or increasing PA levels during pregnancy.

Similarly, Jiang et al. ${ }^{[20]}$ showed that PA levels during pregnancy, as well as pre-pregnancy BMI are inversely associated with gestational weight gain. Using pedometry to measure PA, they showed that the sedentary group ( $<5000$ steps per day) gained $1.45 \mathrm{~kg}$ ( $8 \%$ of the total GWG for the sedentary group) more weight during the last two trimesters of pregnancy than the active group $(>10000$ steps per day). ${ }^{[20]}$ In addition, a recent review has highlighted the beneficial effect of PA in reducing the risk of pre-eclampsia, ${ }^{[21]}$ possibly via its role in weight management.

Furthermore, Dempsey et al. ${ }^{[22]}$ have shown that PA during pregnancy is associated with a $60 \%$ lower incidence of GDM in women who were active before and during pregnancy. In addition to reducing the risk of developing of gestational diabetes, Bung and $\operatorname{Artal}^{[23]}$ also found that exercise has a critical role to play once GDM has been diagnosed, potentially resulting in avoiding insulin therapy. Similarly, a longitudinal study by Van Poppel et al. ${ }^{[24]}$ involving measurements of time spent in moderate-to-vigorous physical activity measured at 15,24 and 32 weeks of gestation concluded that at-risk pregnant women should be encouraged to increase their PA levels for improved insulin and triglyceride responses. 


\section{Patterns of physical activity during pregnancy}

Despite the benefits of regular physical activity during pregnancy, ${ }^{[18]}$ data from the US National Health and Nutrition Estimation Survey (NHANES) has shown that the majority of pregnant women are not sufficiently physically active, tending to do less PA than their nonpregnant counterparts, or they reduce their PA during pregnancy. ${ }^{[25]}$

Epidemiological research suggests that $40-56 \%$ of pregnant women in the United States participate in recreational activity during pregnancy. Data from the women in this study report that the majority of PA in pregnant women comes from domestic responsibilities and childcare. ${ }^{[25]}$ Although little is known about PA patterns during pregnancy in lower-and-middle income (LMIC) countries, a recent study conducted on South African women presenting at two public health clinics in Cape Town utilised the Global Physical Activity Questionnaire to assess PA and its domains. The study showed that women meeting PA guidelines participated in significantly more activity at work and walking for transport than the inactive group. There was very little activity in the leisure domain in both groups. In the same study, average total sitting time (as shown in the GPAQ), and excluding time sleeping, for this group of convenientlysampled, pregnant South African women was significantly higher in the group not meeting PA guidelines, than in the sufficiently active group $(13.0 \pm 3.1 \mathrm{hrs} /$ day vs. $9.1 \pm 3.7 \mathrm{hrs} /$ day, $\mathrm{p}=0.004)$. Similarly, overall sedentary time was higher in the inactivity group $(\mathrm{p}=0.003)$ than in the active group $(14.9 \pm 4.0 \mathrm{hrs} /$ day vs. $10.3 \pm 4.2 \mathrm{hrs} /$ day, respectively).

Furthermore, research on prenatal PA patterns shows that all levels of PA tend to decrease during pregnancy, and physical activities tend to give way to more sedentary behaviours, including watching television and reading. ${ }^{[25]}$ NHANES data on sedentary behaviour during pregnancy have shown that pregnant women in the US spend up to five hours per day watching television, and that sedentary time during pregnancy has been positively associated with increased maternal body weight. ${ }^{[25]}$ In addition, there may be foetal consequences of maternal inactivity. For example, in the Avon Longitudinal Study of Parents and Children (ALSPAC), sedentary pregnant women were at a greater risk of giving birth to low birth weight babies, than their more active counterparts. ${ }^{[2]}$ Therefore although physical activity may have this protective effect, it appears to decline during pregnancy, especially in the leisure domain.

\section{Factors influencing physical activity during pregnancy}

Several maternal and socio-economic characteristics which influence PA levels have been identified. For example, younger maternal and gestational age have been shown to influence the amount and type of PA. In addition, a lower maternal BMI and better overall health status, is associated with greater activity levels. ${ }^{[25]}$

Socio-economic factors, such as a higher education level, social class and income, have all been associated with increased participation in PA during pregnancy ${ }^{[6,25]}$ Parity has also been shown to be associated with PA levels amongst pregnant women. Evenson et al. ${ }^{[6]}$ found that women who have three or more children are less likely to engage in leisure time PA than women with less than three children. Conversely, Liu et al. ${ }^{[27]}$ found that multiparous women are more likely to engage in strenuous activity for at least three hours per week, when compared to nulliparous women. Although the authors do not specify the domain of activity, it is possible that this PA is related to child-care activities and domestic work.
Since international studies have a very different social and environmental context to South Africa, factors which influence physical activity participation are also likely to be different in South African women. A recent unpublished study (Pearson, Micklesfield and Lambert, 2013 - Honours dissertation) has shown that South African women are more likely to exercise if they have someone to exercise with and somewhere they could go to in order to exercise. The most commonly reported barriers to physical activity during pregnancy appeared to be painful knees, back or abdomen, tiredness and no time to exercise. Although the current evidence helps to give a broad idea of factors which may influence PA participation during pregnancy, it is necessary for more research to be done in the South African context in order to support the evidence found in the study described above, since this is the only known source of such information for South African women. This information pertaining to correlates of PA among South African women will allow the development of effective interventions relating to PA during pregnancy.

In addition to understanding correlates and patterns of physical activity and sedentary behaviour, it is also essential to understand attitudes and perceptions towards PA in pregnancy. Despite the evidence that supports the benefits of PA during pregnancy, many pregnant women frequently report that they are unsure of the safety of exercising during pregnancy and thus reduce their PA after becoming pregnant. ${ }^{[28]}$ In a recent study done in South Africa, Muzigaba et al. ${ }^{[28]}$ found that although South African women are interested in participating in PA during pregnancy, many were unaware of what is safely recommended. In addition, the women reported a lack of information and advice from healthcare providers, which may explain their safety concerns for themselves and their unborn baby. Women also frequently report discomfort, lack of free time, tiredness, pain and lack of social support as reasons for not engaging in PA during pregnancy. ${ }^{[28]}$

\section{Conclusion}

In conclusion, the burden of NCDs and obesity in South African women is increasing and placing immense pressure on the healthcare system. Due to the role of the intrauterine environment on offspring health, pregnancy has been identified as one of the important periods in life to influence the health of both the mother and child. PA has been shown to be an important factor which may help to improve pregnancy outcomes. However, most of the literature examining PA trends during pregnancy comes from international studies, with limited data on South African women. As such, it is imperative that the PA levels, as well as the beliefs, barriers and facilitators of PA during pregnancy are better understood in a South African context. This would assist in informing public health intervention strategies to reduce the prevalence of NCDs and obesity for women and their offspring.

\section{Conflict of interest. None declared.}

\section{References}

1. Mayosi BM, Flisher AJ, Lalloo UG, et al. The burden of non-communicable diseases in South Africa. Lancet 2009;374(9693):934-947. [http://dx.doi.org/10.1016/S01406736(09)61087-4]

2. Baleta A, Mitchell F. Country in Focus: diabetes and obesity in South Africa. Lancet Diabetes Endocrinol 2014;2(9):687-688. [http://dx.doi.org/10.1016/S22138587(14)70091-9] 
3. Lee I-M, Shiroma EJ, Lobelo F, et al. Effect of physical inactivity on major noncommunicable diseases worldwide: an analysis of burden of disease and life expectancy. Lancet 2012;380(9838):219-229. [http://dx.doi.org/10.1016/S0140 6736(12)61031-9]

4. Dickie K, Micklesfield LK, Chantler S, et al. Meeting physical activity guidelines is associated with reduced risk for cardiovascular disease in black South African women; a 5.5-year follow-up study. BMC Public Health 2014 May 23;14:498. [http:// dx.doi.org/10.1186/1471-2458-14-498]

5. Oken E, Gillman MW. Fetal origins of obesity. Obes Res 2003;11(4):496-506. [http:// dx.doi.org/10.1038/oby.2003.69]

6. Evenson KR, Savitz D, Huston SL. Leisure-time physical activity among pregnan women in the US. Paediatr Perinat Epidemiol 2004;18(6):400-407. [http://dx.doi. org/10.1111/j.1365-3016.2004.00595.x]

7. Unwin N, Mugusi F, Aspray T, et al. Tackling the emerging pandemic of noncommunicable diseases in sub-Saharan Africa. Public Health 1999;113(3):141-146. [http://dx.doi.org/10.1038/sj.ph.1900549]

8. Bourne LT, Lambert EV, Steyn K. Where does the black population of South Africa stand on the nutrition transition? Public Health Nutr 2002;5(1A):157-162. [http:// dx.doi.org/10.1079/PHN2001288]

9. Kruger HS, Venter CS, Vorster HH. Obesity in African women in the North West Province, South Africa is associated with an increased risk of non-communicable diseases: the THUSA study. Br J Nutr 2001;86(06):733. [http://dx.doi.org/10.1079/ BJN2001469]

10. Galtier-Dereure F, Boegner C, Bringer J. Obesity and pregnancy: complications and cost. Am J Clin Nutr 2000;71(5 Suppl):1242S-8S. [http://www.ncbi.nlm.nih.gov/ pubmed/10799397]

11. Guelinckx I, Devlieger R, Beckers K, et al. Maternal obesity: pregnancy complications, gestational weight gain and nutrition. Obes Rev 2008;9(2):140-150. [http://dx.doi. org/10.1111/j.1467-789X.2007.00464.x]

12. Lindheimer MD. Hypertension in pregnancy [clinical conference]. Hypertension 1993;22(1):127-137. [http://dx.doi.org/10.1161/01.HYP.22.1.127]

13. Moodley J. Maternal deaths associated with hypertension in South Africa : lessons to learn from the Saving Mothers report, 2005-2007. Cardiovasc J Afr 2011;22(1):31-35. [http://dx.doi.org/10.5830/CVJA-2010-042]

14. Macaulay S, Dunger DB, Norris SA. Gestational diabetes mellitus in Africa: a systematic review. PLoS One 2014;9(6):e97871. [http://dx.doi.org/10.1371/journal. pone.0097871]

15. Catalano PM, Kirwan JP, Mouzon SH, et al. Gestational Diabetes and Insulin Resistance: Role in Short- and Long-Term Implications for Mother and Fetus. J Nutr 2003;133:1674-1683.

16. Drake A, Walker B. The intergenerational effects of fetal programming: nongenomic mechanisms for the inheritance of low birth weight and cardiovascular risk J Endocrinol 2004;180(1):1-16. [http://www.ncbi.nlm.nih.gov/pubmed/14709139]

17. Gavard J, Artal R. Effect of exercise on pregnancy outcome. Clin Obstet Gynecol 2008;51(2):467-480. [http://dx.doi.org/10.1097/GRF.0b013e31816febld]
18. Clapp JF. Exercise during pregnancy. A clinical update. Clin Sports Med 2000;19(2) 273-286. [http://www.ncbi.nlm.nih.gov/pubmed/10740759] Accessed April 7, 2015.

19. Olson CM, Strawderman MS, Hinton PS, et al. Gestational weight gain and postpartum behaviors associated with weight change from early pregnancy to $1 \mathrm{y}$ postpartum. Int J Obes Relat Metab Disord 2003;27(1):117-127. [http://dx.doi. org/10.1038/sj.ijo.0802156]

20. Jiang H, Qian X, Li M, et al. Can physical activity reduce excessive gestational weigh gain? Findings from a Chinese urban pregnant women cohort study. Int J Behav Nutr Phys Act 2012;9:12. [http://dx.doi.org/10.1186/1479-5868-9-12]

21. Aune D, Saugstad OD, Henriksen T, et al. Physical Activity and the Risk of Preeclampsia. Epidemiology 2014;25(3):331-343. [http://dx.doi.org/10.1097/ EDE.0000000000000036]

22. Dempsey JC, Butler CL, Sorensen TK, et al A case-control study of maternal recreational physical activity and risk of gestational diabetes mellitus. Diabetes Res Clin Pract 2004;66(2):203-215. [http://dx.doi.org/10.1016/j.diabres.2004.03.010]

23. Bung P, Artal R. Gestational diabetes and exercise: a survey. Semin Perinato 1996;20(4):328-333. [http://www.ncbi.nlm.nih.gov/pubmed/8888458] Accessed September 8, 2013

24. Van Poppel MNM, Oostdam N, Eekhoff MEW, et al. Longitudinal relationship of physical activity with insulin sensitivity in overweight and obese pregnant women. J Clin Endocrinol Metab 2013;98(7):2929-2935. [http://dx.doi.org/10.1210/jc.20131570]

25. Evenson KR, Wen F. National trends in self-reported physical activity and sedentary behaviors among pregnant women: NHANES 1999-2006. Prev Med (Baltim) 2010; 50(3):123-128. [http://dx.doi.org/10.1016/j.ypmed.2009.12.015]

26. Both MI, Overvest M, Wildhagen MF, et al. The association of daily physical activity and birth outcome: a population-based cohort study. Eur J Epidemiol 2010;25(6): 421-429. [http://dx.doi.org/10.1007/s10654-010-9458-0]

27. Liu J, Blair SN, Teng Y, et al. Physical activity during pregnancy in a prospective cohort of British women: results from the Avon longitudinal study of parents and children. Eur J Epidemiol 2011;26(3):237-247. [http://dx.doi.org/10.1007/s10654010-9538-1]

28. Muzigaba M, Kolbe-Alexander TL, Wong F. The Perceived Role and Influencer of Physical Activity Among Pregnant Women From Low Socioeconomic Status Communities in South Africa. J Phys Act Heal 2014;11(7):1276-1283. [http://dx.doi org/10.1123/jpah.2012-0386]

* References had to be reduced because of constraints on space. A comprehensive list can be obtained from the corresponding author. 